\title{
The dual-task SRT procedure: Fine-tuning the timing
}

\author{
ANDREW T. HSIAO and ARTHUR S. REBER \\ Brooklyn College and The Graduate Center of CUNY, Brooklyn, New York
}

\begin{abstract}
In the standard sequential reaction time study, subjects are presented with a repeating sequence of targets to which they must respond as rapidly as possible. With practice reaction times decrease, suggesting a learned ability to exploit the repeating patterns in the display. In a common variation, a second task is interposed, typically a tone-counting task in which subjects must keep track of the frequency with which particular tones occur. In the canonical experiment, these tones appear at varying times in the interval between the subjects' response to a target and the next target. Data are presented that show that this latter variable (the RSOA, response-secondary stimulus onset asynchrony) actually plays an important but previously hidden role in these experiments. A model based on an extension of the notion of the psychological refractory period is introduced to explain these findings.
\end{abstract}

The sequential reaction time (or SRT) task was introduced by Nissen and her colleagues just over a decade ago (Nissen \& Bullemer, 1987); since then, a virtual cottage industry has sprung up around it. There are many reasons for the procedure's popularity. The task, at least on the surface, is simplicity itself. Subjects sit in front of a monitor on which a number of locations are marked (usually four but occasionally five or six). A series of target stimuli (typically an * or another symbol) appear at the locations. The subject's task is to press the key that corresponds spatially with the location of each target as quickly and accurately as possible. After each response there is a short interval before the next target appears.

In the canonical procedure, the sequence of target locations has some degree of regularity. In Nissen and Bullemer's original (1987) study and in most of those run since, a repeating sequence with particular statistical properties is used, although several experiments have used a nonrepeating sequence in which a complex set of rules dictates successive locations (Baldwin \& Kutas, 1997; Cleeremans \& McClelland, 1991; Hsiao \& A. S. Reber, 1998; Jiménez, Mendez, \& Cleeremans, 1996). Subjects are generally run through a series of blocks of roughly 100 trials with the experiment usually comprising anywhere from 7 to 17 such blocks.

The classic finding is straightforward and remarkably robust. Reaction times (RTs) decrease as subjects learn to exploit the structure in the target sequence. Learning is evaluated either by comparing RTs with those of control subjects working with nonrepeating or unstructured

Support for this project came from a PSC-CUNY grant. We thank Simon Rah and Lieb Litman for help on various phases of this project. The data from this study were reported at the 1997 meeting of the Psychonomic Society. Correspondence concerning this article should be addressed to A. S. Reber, Department of Psychology, Brooklyn College of CUNY, Brooklyn, NY 11210 (e-mail: areber@brooklyn.cuny.edu). sequences or by introducing one or more transfer blocks where the sequence is modified in some fashion, and any increase in RTs is noted.

In a commonly employed variation, a secondary task is introduced. In this "dual-task" experiment, somewhere during the interval between the keypress and the next target either a high- or low-pitched tone sounds. In addition to responding to each successive target, subjects must also keep an accurate running count of how many, say, highpitched tones occurred in each block of trials.

It takes but a moment's reflection to understand why this procedure has attracted so much attention. First, it is a model environment for the study of implicit learning, for in most of these studies the subjects were unaware of the existence of the structured nature of the sequence (P. J. Reber \& Squire, 1994; Reed \& Johnson, 1994; Willingham, Nissen, \& Bullemer, 1989; but see also Shanks \& Johnstone, 1998, for a different read on this literature). Second, by manipulating various aspects of the tonecounting task, the role of attention in learning can be readily explored (see Hsiao \& A. S. Reber, 1998, for an overview of this work). Third, by tweaking the underlying statistical characteristics of the sequence, the procedure becomes a forum for examining the impact of complexity on learning (Cohen, Ivry, \& Keele, 1990; Stadler, 1989). Finally, because the technique produces a large and reliable data base, it becomes an excellent platform for testing theoretical models of learning and representation (Cleeremans, 1993; Cleeremans \& Jiménez, 1998; Keele, Ivry, Hazeltine, Mayr, \& Heuer, 1999) as well as for exploring the neuroanatomic underpinnings of implicit learning (Baldwin \& Kutas, 1997; Curran, 1998; Eimer, Goschke, Schlaghecken, \& Stürmer, 1996; Grafton, Hazeltine, \& Ivry, 1995; Keele et al., 1999).

As the task itself has become better understood, it has, perhaps predictably, begun to reveal surprisingly subtle and psychologically significant nuances that have been 
glossed over in the earlier work. For example, the original conception of the secondary tone-counting variation was that the dual-task environment compromised learning of the target sequence because limited attentional resources were being diverted by the need to satisfy the demands of the other task. But studies by Mayr (1996), Rah, A. S. Reber, and Hsiao (2000), and Schmidtke and Heuer (1997) all point to the conclusion that the tone-counting task is not so much a "secondary" task as an additional source of potential covariations in a complex, multifaceted environment. Both Rah et al. and Schmidtke and Heuer built in contingencies between the tones and the targets and found that, under these conditions, the tone-counting task no longer compromises performance. In fact, Rah et al. found that, under some circumstances, the presence of the contingent secondary tones can actually enhance performance.

In addition, recent work (Destrebecqz \& Cleeremans, 1999) suggests strongly that the time between the subject's response to a target and the appearance of the next target has a dramatic effect on the very nature of the learning that takes place. When the next target appears with no lag, learning is virtually completely implicit in nature. When a substantial lag $(250 \mathrm{msec})$ is present, explicit processes tend to dominate.

In this report, we focus on another unnoticed component of the standard dual-task SRT experiment, the responsesecondary stimulus onset asynchrony (RSOA). The standard experiment has the following key features. Each trial begins with the presentation of the target stimulus (T), which stays on until the proper keypress response $(\mathrm{R})$ is made. A short (usually around $200 \mathrm{msec}$ ) interval follows before the next target is presented. In this interval, the "secondary" tone stimulus (S) is presented.

While reviewing the literature we discovered an odd lack of consistency concerning the duration of the secondary tone and its temporal position in the interval between the response and the subsequent target. For example, some researchers (Heuer \& Schmidtke, 1996; Rah et al., 2000; Schmidtke \& Heuer, 1997) used a 100-msec tone that always began $50 \mathrm{msec}$ after the keypress. Others (Stadler, 1995) used a short $(83-\mathrm{msec})$ tone that began almost immediately (e.g., $17 \mathrm{msec}$ ) after the keypress response, but most of the studies (Cohen et al., 1990; Curran \& Keele, 1993; Frensch, Buchner, \& Lin, 1994, among many others) used a tone of often unspecified duration that was randomly presented either 40,80 , or $120 \mathrm{msec}$ after the primary response to the target in an interval of $200 \mathrm{msec}^{1}$

This lack of consistency is of some concern, particularly because the more the microstructure of the SRT task has been examined, the more complexity is revealed. Both we (unpublished data) and Reed and Johnson (1994) have observed fairly large RT effects (i.e., in the 40- to 50 -msec range) produced by seemingly innocent factors. For example, if a sequence contains a single alternation between two locations (e.g., 141 or 232), the RTs to the second occurrence are typically longer than to the first, suggesting that a kind of inhibition of return effect is operating. However, if a location repeats immediately, RTs to the second are routinely shorter, implying that immediate priming can override inhibition of return. In addition, if a sequence contains a "chunk" like 2431 or 4213 , the mean RTs to the final element (or the one "not seen recently") tend to be faster. And, finally, we routinely found that the RTs made with the index fingers tended to be faster than those made with either the middle or ring fingers.

Although these microcognitive elements all have some attraction as possible avenues of exploration, in this experiment, our primary aim was to try to control for them while examining the role of the RSOA. We present data here that show that the chaotic variation of this factor has been masking important psychological processes.

\section{METHOD}

\section{Target Display}

The primary visual targets were presented using the circular display shown in Figure 1. There are five target locations, the four arrows and the center spot. At a comfortable viewing distance of roughly $500 \mathrm{~mm}$, the full display subtends a visual angle of approximately $3.75^{\circ}$. The numerical keypad was used with the " 5 " key for the central spot and "4", " 8 ", " 6 ", and " 2 " keys for the left, up, right, and down arrows, respectively.

All responses to the targets were made using a single finger. Subjects could use any finger they wanted so long as they continued to use it for the entire experiment. They were monitored to make sure that they conformed to instructions.

\section{The Target Sequence}

A repeating 12-element sequence (LCURLDRCLRUD, where $\mathrm{L}=$ left, $\mathrm{C}=$ center, $\mathrm{U}=$ up, etc.) was used. Note that this sequence contains no immediate repeats and no single alternations.

\section{The Secondary Tones}

The high- and low-pitched tones were $850 \mathrm{~Hz}$ and $550 \mathrm{~Hz}$, respectively, and occurred randomly. The subjects were asked to keep track of either the high- or low-pitched tones and to report the number detected at the end of each block of trials. Each trial was based on a 200-msec interval between the subject's keypress response (R) to a target and the appearance of the next target. The secondary tone stimulus (S) came on either 50, 100, or $150 \mathrm{msec}$ after the subject's response and lasted for $50 \mathrm{msec}$. Thus, the main independent variable in the experiment was the RSOA.

\section{Procedure}

Learning consisted of eight blocks of 120 trials each. Block 9 was a transfer block during which subjects were presented with a quasi-random series of 120 targets; the repeating sequence was restored on Block 10. The target sequence on the transfer block was controlled so that it contained the same frequency of occurrence of individual targets and matched as closely as possible the first-order contingencies found in the repeating sequence. See Table 1 for details on the match between the learning and transfer sequences. Note that the use of a transfer block with these properties ensures that any learning that emerges must be fairly deep and depend on the detection of at least second-order conditionals (SOCs).

Learning was assessed by comparing the RTs from Blocks 8 and 10 with those from Block 9 . Since learning was not complete by the end of Block 8 , this comparison is the appropriate one since it shows the extent to which the semirandom events on Block 9 disrupted learning. 


\section{Primary Stimulus Display}

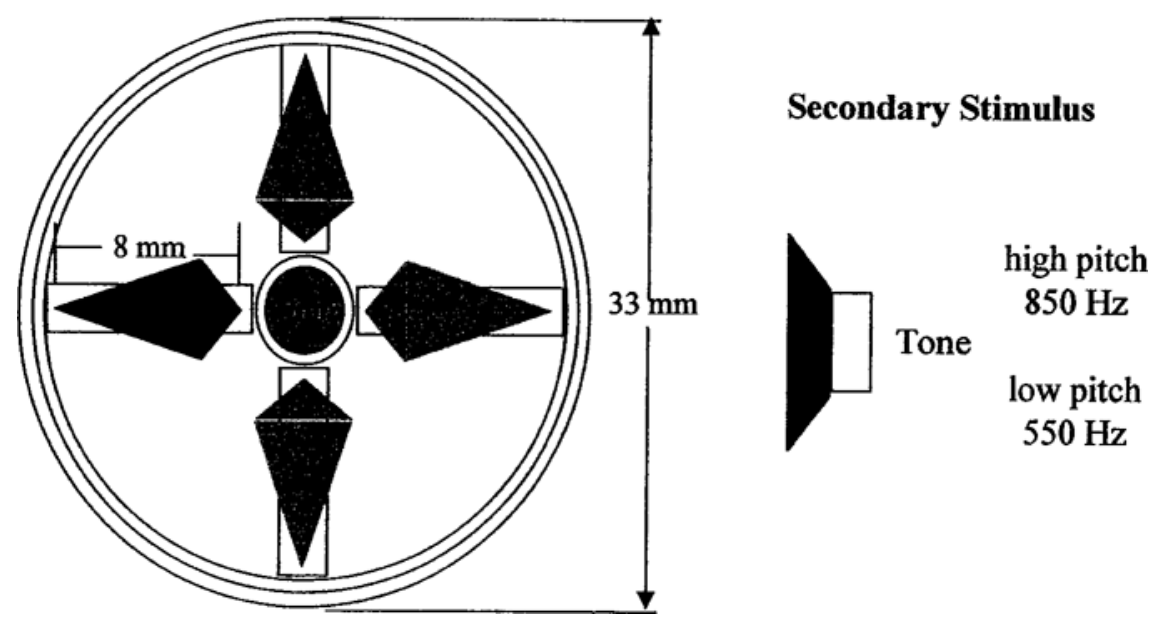

Figure 1. A schematic outline of the display with a layout of the response keys.

\section{Subjects}

The experimental subjects were 249 undergraduates at Brooklyn College, all of whom were enrolled in Introductory Psychology and received course credit for participating. One third were run with each of the three RSOAs.

Poor counters, good counters, and estimators. In the standard dual-task SRT experiment, subjects who fail to perform to criteria are eliminated from analysis. Specifically, those with high error rates on the primary target task are typically dropped, on the grounds that they are not following instructions; those with high error rates on the tone-counting task are eliminated because they are likely not to be paying attention to the tones. We dropped 24 subjects whose error rate on the target task exceeded $5 \%$ but, for reasons that will become clear, we retained a total of 121 subjects with high error rates (i.e., more than $10 \%$ ) on the tone-counting task. We have found (Rah et al., 2000) that these "poor counters" are useful as a natural control group. They appeared not be expending much in the way of attentional resources on the secondary task and performed very differently from "good counters." Consequently, there are three a posteriori groups of poor counters (one for each RSOA).

Finally, we ran three groups of "estimators." These subjects were selected at random from the same pool and run in identical fashion except that, instead of counting the number of, say, high-pitched tones in each trial block, they were asked for an estimate of how many they thought had sounded. As above, the subjects in this group whose error rate on the primary task exceeded $5 \%$ were dropped from analysis. After excluding the high-error rate subjects and partialling out the good and poor counters, we ended up with a total of 225 subjects distributed as shown in Table 2.

\section{Design}

The experiment, thus, was a 3 (good counters, poor counters, and estimators) $\times 3$ (50-, 100-, and 150-msec RSOAs) design.

\section{Results}

Distinguishing the groups. First, appreciate how differently the 41 subjects who qualified as good counters approached the task compared with the others. Although we used a $10 \%$ error rate as the criterion to differentiate good from poor counters, we actually have two dramatically different groups. The good counters clearly made every effort to follow instructions. Their overall error rate was only $5.7 \%(S D=2.31 \%)$ compared with the poor counters' mean error rate of $56.7 \%(S D=29.6 \%)$. The estimators, perhaps not surprisingly, look a lot like the poor counters, with an error rate of $65.9 \%(S D=29.1 \%)$.

Table 1

Match Between the Simple Frequency of Occurrence of Locations and First-Order Conditionals for the Learning and Transfer Sequences

\begin{tabular}{|c|c|c|c|c|c|}
\hline \multicolumn{6}{|c|}{ Simple Event Frequencies (per block of 120 trials) } \\
\hline & $\mathrm{Up}$ & Down & Left & Right & Center \\
\hline Learning sequence & 20 & 20 & 30 & 30 & 2 \\
\hline Transfer sequence & 20 & 20 & 30 & 30 & 2 \\
\hline
\end{tabular}

First-Order Conditional Frequencies (per block of 120 trials)

\begin{tabular}{lrrrrrrrrrrrr} 
& UD & UR & DL & DR & LD & LR & LC & RU & RL & RC & CU & CL \\
\hline Learning sequence & 10 & 10 & 9 & 10 & 10 & 10 & 10 & 10 & 10 & 10 & 10 & 10 \\
Transfer sequence & 12 & 8 & 10 & 10 & 8 & 12 & 10 & 9 & 11 & 10 & 11 & 8 \\
\hline
\end{tabular}

Note-The learning sequence was LCURLDRCLRUD; U = Up, D = Down, L = Left,

$\mathrm{R}=\mathrm{Right}$, and $\mathrm{C}=$ Center. See Figure 1 for specifics of the display. Thus, for example, the subsequence Up-Down occurred exactly 10 times in each sequence during learning and exactly 12 times during transfer. 
Table 2

Final Numbers of Subjects in Each of the RSOA Conditions After Excluding Those With Error-Rates on the Primary Target Task Greater Than 5\%

\begin{tabular}{lcccr}
\hline & \multicolumn{4}{c}{ RSOA } \\
\cline { 2 - 5 } \multicolumn{1}{c}{ Group } & $50 \mathrm{msec}$ & $100 \mathrm{msec}$ & $150 \mathrm{msec}$ & Total \\
\hline Good counters & 14 & 15 & 12 & 41 \\
Poor counters & 40 & 41 & 40 & 121 \\
Estimators & 21 & 20 & 22 & 63 \\
Totals & 75 & 76 & 74 & 225 \\
\hline
\end{tabular}

It is the close attention that the good counters paid to the task that allows us to unpack the role of the RSOA. In passing, note that the accuracy on the primary target task was unaffected by whether Ss were diverting attentional resources to the secondary task. The overall accuracy rates were $98.7 \%, 97.8 \%$, and $98.3 \%$ for good counters, poor counters, and estimators, respectively.

Learning. Even though we tried to reduce the amount of variance in the experiment by introducing a number of controls, our data were still plagued by the high subjectto-subject and trial-to-trial variability that characterizes these SRT tasks. Consequently, all following analyses were carried out using the median RT of each subject for each cycle of the sequence and then calculating the mean of those medians for the block.

The extent to which the subjects learned about the repeating structure of the target sequence is given by the extent to which the introduction of the nonrepeating semirandom sequence on Block 9 disrupted performance. Figure 2 presents the means of medians for the three groups (collapsed across RSOAs) for the full 10 blocks of the experiment. Two effects are apparent. First, overall RTs were systematically related to the manner in which subjects approached the task. Good counters were slow, estimators were fast, and the poor counters lie in between. The poor counters and estimators did not differ statistically from each other, although both were significantly faster than the good counters (Bonferroni tests, $p<.05$ in both cases).

However, although these groups differ with respect to overall RT, all three showed learning in that the RTs on Block 9 were significantly longer than the average of Blocks 8 and $10[t \mathrm{~s}=12.1$ for the good counters $(d f=40)$, 42.5 for the poor counters $(d f=120)$, and 47.8 for the estimators $(d f=62)$; all $p \mathrm{~s}<.001]$. The mean difference between Block 9 and the means of Blocks 8 and 10 was $24.2 \mathrm{msec}$ for the good counters, $41.6 \mathrm{msec}$ for the poor counters, and $40.7 \mathrm{msec}$ for the estimators. The pattern here is the same as for the overall RTs. The overall group effect is significant $\left[F(2,222)=5.18, M S_{\mathrm{e}}=2,640.4, p<\right.$ .006]. Poor counters and estimators did not differ from each other, but both showed significantly better learning than the good counters (Bonferroni tests, $p<.05$ in both cases).

None of this is new or particularly surprising. It is well known that an attention-demanding secondary task compromises learning in these types of experiments. The intriguing finding emerges when the good counters are compared directly with the poor counters. Here, in addition to the simple group differences, a significant group $\times$ RSOA interaction was found. $\left[F(2,156)=3.40, M S_{\mathrm{e}}=\right.$ $34,320.9, p<.03$ ]. Figure 3 shows the learning effect (as given by the mean difference between the RTs on Block 9 minus the means of Blocks 8 and 10) for each of the three RSOAs for all three groups. As one might expect, the temporal location of the tone had no systematic impact on the estimators or on the poor counters. After all, these subjects were essentially ignoring the secondary tones. However, in the case of the good counters, learning of the underlying structure of the sequence was systematically related to the RSOA. When the secondary tone occurred early in the interval, we observed learning that was in close proximity to that seen under all RSOAs for the other groups. When the RSOA was in the middle of the interval $(100 \mathrm{msec})$, the learning effect dropped and,

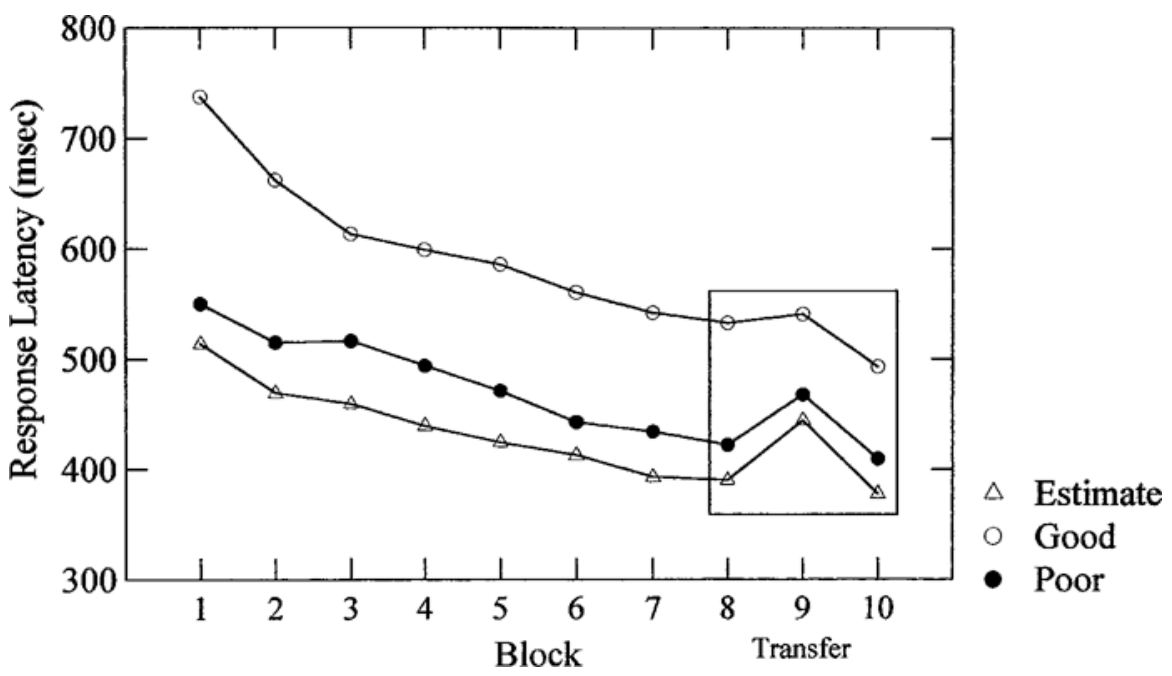

Figure 2. Means of median RTs over the 10 trial blocks for the three subject groups collapsed across RSOAs. 


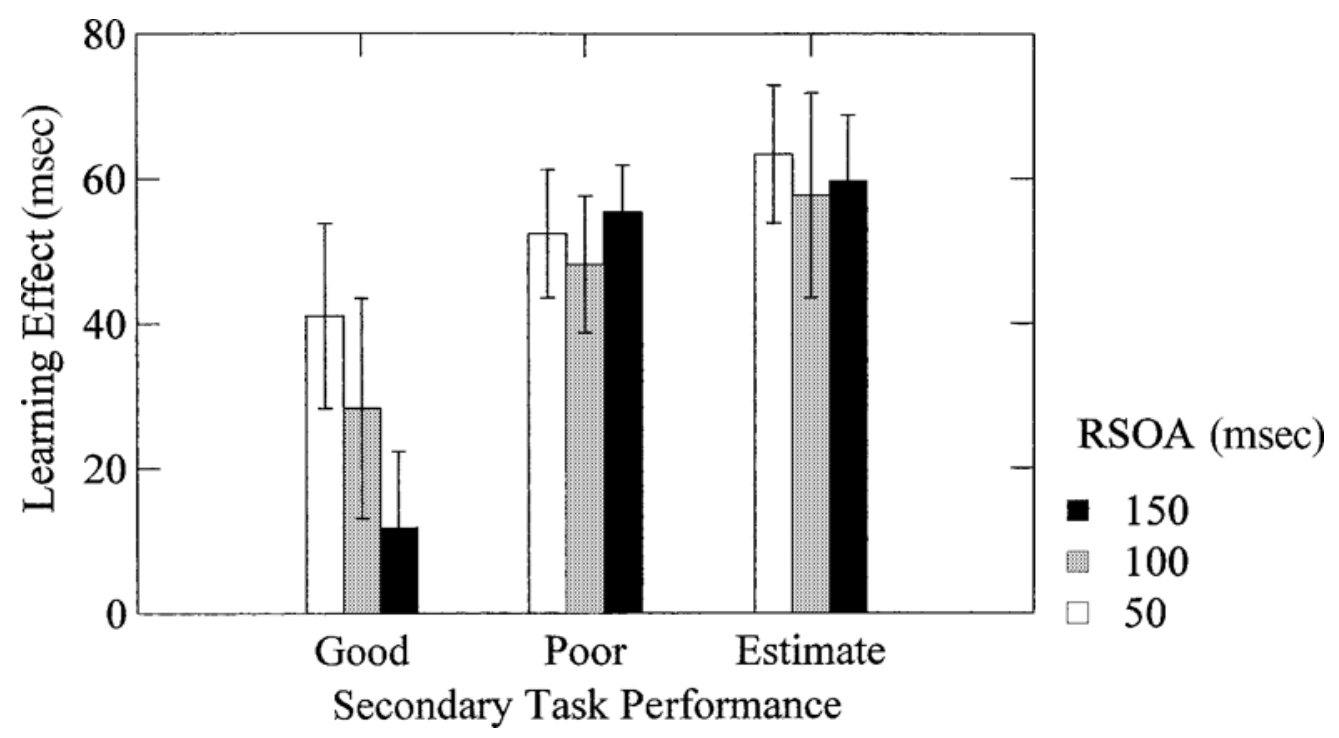

Figure 3. The learning effect size for each group and RSOA.

when it occured toward the end $(150 \mathrm{msec})$, learning was essentially eliminated.

Finally, there was a hint of an additional impact of the temporal placement of the secondary tone stimuli in that the good counters with the 50-msec RSOAs tended to display the fastest response times (see Figure 4). Although the pattern is suggestive, the 50-msec group was not reliably different from the other RSOAs $(p=.096)$ when compared with the $150-\mathrm{msec}$ subjects. We suspect that part of the problem here was lack of statistical power owing to the small number of good counters who passed the strict criterion we imposed.

\section{DISCUSSION}

The protocol used in the classical dual-task SRT study may appear simple on the surface, but it is fraught with complexities. Our subjects were asked to carry out a rather complex task that involved attending and responding to two ongoing sequences. The first was made up of a series of visual targets following a repeating pattern; the second comprised a series of auditory stimuli that were not easy to distinguish, were devoid of structure, and were unrelated to the visual targets. Successful performance on this task required that, on each trial $(n)$, subjects must detect the location of the target $\left(\mathrm{T}_{n}\right)$, respond to it quickly and reliably $\left(\mathrm{R}_{n}\right)$, process the secondary tone $\left(\mathrm{S}_{n}\right)$ that followed the keypress by some RSOA, determine its identity and, if appropriate, upgrade the count accurately, and then react to the subsequent visual target $\left(\mathrm{T}_{n+1}\right)$. Because the transfer sequences were matched for both zeroand first-order conditionals, learning of the primary target sequence required a hierarchical encoding, involving integration across a minimum of three segments of the sequence.
We report two key findings. First, the secondary tonecounting task, when treated seriously as an element in this experiment (i.e., the good counters), dramatically slowed down responding to the visual targets. In and of itself, the occurrence of these tones did not eliminate learning of the sequence since even the good counters showed reliable learning of the sequence as evidenced by significantly elevated RTs during the transfer block. However, for the subjects who diverted resources to the secondary auditory display, the temporal location of the tone stimulus within the $\mathrm{R}_{n}-\mathrm{T}_{n+1}$ interval had a strong impact on performance. The later in the $\mathrm{R}_{n}-\mathrm{T}_{n+1}$ interval $S_{n}$ occurs, the more it compromises subjects' performance on the primary visual target task.

This pattern of behavior is reminiscent of, although not strictly concordant with, that observed in studies on the psychological refractory period (PRP). In these studies, whenever subjects are required to perform two tasks within close temporal proximity, the response to the second task is compromised in some fashion (usually slowed, since RTs are the standard measure of these effects) by the need to process and respond to the first (see Pashler, 1998, especially Chapter 6 for a review). This reduction in performance is generally regarded as resulting from a kind of bottleneck effect in which the various processes that need to accompany successful response to the second stimulus (detection, identification, and preparation) get choked off by the processing of the first. Relevantly, the PRP has been shown to manifest itself even when the two input stimuli come from distinct sensory modalities (Creamer, 1963; Jolicoeur, 1999).

In the critical groups in our experiment, the good counters with the differing RSOAs, the two events that are analogous to the canonical PRP experiment are the cluster of processing responses to $S_{n}$ and the keypress to the sub- 


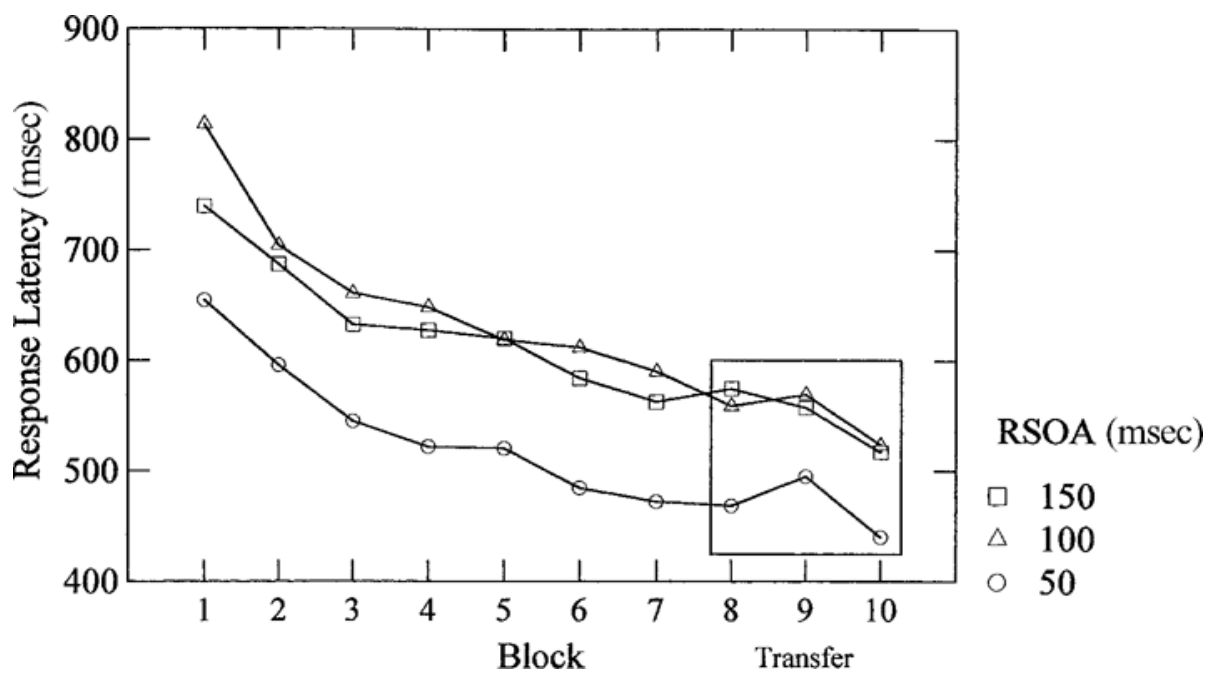

Figure 4. Means of median RTs from the good counters across blocks for the three RSOAs.

sequent $T_{n+1}$. Our situation, of course, is different from that traditionally used when exploring the PRP. First, our subjects did not respond to each $S_{n}$ with a simple keypress. Their obligations were considerably more complex. They had to detect each tone, determine its identity, upgrade (or not) the ongoing count, and detect any possible covariations of the tone sequence and the successive primary targets-a series of perceptual and cognitive operations with obvious attentional and temporal demands. Interestingly, our data suggest that such a complex set of operations can still be carried out in roughly $50-100 \mathrm{msec}$. Moreover, the second response our subjects had to make, the keypress to the subsequent $\mathrm{T}_{n+1}$, was also on a different level from that in the standard PRP task. The subjects in our study had not only to respond to each $\mathrm{T}_{n}$, they had to detect patterns of covariations between $\mathrm{T}_{n-1}$, $\mathrm{T}_{n}$, and $\mathrm{T}_{n+1}$, since learning in this SRT task required detection of at least second-order conditionals.

The overall slowing of RTs seen in Figure 2 falls neatly out of an uncomplicated PRP interpretation. We would expect the cascade of responses that are recruited by the secondary tone to produce a general slowing of the responses to the subsequent visual target. The hinted differential slowing of overall RT shown in Figure 4, if it is ultimately shown to be reliable, also fits comfortably with Pashler's (1998) theory. And, interestingly, it suggests that this sequence of responses to the tones is more or less complete after some 100 to $150 \mathrm{msec}$.

The intriguing finding, the interaction between attention (good counters vs. poor counters and estimators) ${ }^{2}$ and RSOA, requires an extension of the usual interpretation of the PRP. Rather than seeing a general slowing of RTs due to the interposed tones, we observed a differential impact on the capacity of the subjects to encode the deep structure (i.e., at least to the level of second-order conditionals) of the target sequence. It appears that during the roughly 100 to $150 \mathrm{msec}$ that the PRP appears to last in this experimental setting, the subjects' capacity to detect patterns of covariations in the sequence of target locations was also compromised.

Exactly how this disruption in performance is manifested is not clear, at least not from the models currently entertained. As we noted elsewhere (Hsiao \& Reber, 1998), there are several coherent theoretical interpretations of the impact of the secondary task in the standard SRT experiment. They range from Keele and colleagues' (Curran \& Keele, 1993; Keele et al., 1999) dual-mechanism theory that argues that the secondary task only disrupts the hierarchical encoding mechanism to Stadler's (1995) perspective that emphasizes the role of chunking and grouping of subcomponents of the sequence to Frensch and coworkers' (Frensch, Lin, \& Buchner, 1998; Frensch $\&$ Miner, 1994) contention that the secondary task reduces available short-term memory.

It seems fairly clear that none of these models can account for the findings we present here, simply because none of them contains any mechanism that is sensitive to the variation in the RSOA. The notion of a psychological refractory period helps, but even that adjustment is insufficient. In order to account for the varying impact of the RSOA on sequence learning we need to introduce an additional theoretical construct, a limited-capacity working memory (WM), specifically, one that acts like a pushdown stack. The inclusion of a working-memory component is, of course, not new. Each of the models noted above assumes, in one format or another, a limited-capacity, short-term memory system. In fact, it is difficult to understand how SOC sequences could be learned with a system that does not have a working memory that could hold at least three elements.

The novel element in our model is the presumed pattern of interaction between the psychological refractory period and working memory. As each new visual target occurs, it gets logged in WM and, so long as it is present 
in the stack (order needs to be preserved so that conditionalized representations can be maintained), it is available to be associated with subsequent targets. However, while subjects are engaged in the complex set of operations recruited by the secondary tone (that is, the PRP is still "running"), working memory is taxed, and its effective capacity reduced. Representations of earlier targets get pushed out, become unavailable to be associatively linked with the subsequent targets and, consequently, sequence learning is compromised, perhaps even eliminated. The degree of disruption of performance will, in principle, be dependent on the temporal parameters that affect the PRP, in short, the PRP's "half-life."

\section{REFERENCES}

Baldwin, K. B., \& Kutas, M. (1997). An ERP analysis of implicit structured sequence learning. Psychophyiology, 34, 74-86.

Cleeremans, A. (1993). Mechanisms of implicit learning: Connectionist models of sequence processing. Cambridge, MA: MIT Press.

Cleeremans, A., \& Jiménez, L. (1998). Implicit sequence learning: The truth is in the details. In M. A. Stadler \& P. A. Frensch (Eds.), Handbook of implicit learning (pp. 323-364). Thousand Oaks, CA: Sage.

Cleeremans, A., \& McClelland, J. L. (1991). Learning the structure of event sequences. Journal of Experimental Psychology: General, 120, 235-253.

Cohen, A., Ivry, R. I., \& Keele, S. W. (1990). Attention and structure in sequence learning. Journal of Experimental Psychology: Learning, Memory, \& Cognition, 16, 17-30.

Creamer, L. R. (1963). Event uncertainty, psychological refractory period, and human data processing. Journal of Experimental Psychology, 66, 187-194.

CurRAN, T. (1998). Implicit sequence learning from a cognitive neuroscience perspective: What, how and where? In M. A. Stadler \& P. A. Frensch (Eds.), Handbook of implicit learning (pp. 365-400). Thousand Oaks, CA: Sage.

Curran, T., \& Keele, S. W. (1993). Attentional and nonattentional forms of sequence learning. Journal of Experimental Psychology: Learning, Memory, \& Cognition, 19, 189-202.

Destrebecqz, A., \& Cleeremans, A. (1999). Can sequence learning be implicit? New evidence with the process dissociation procedure. Unpublished manuscript.

Eimer, M., Goschke, R., Schlaghecken, F., \& Stürmer, B. (1996). Explicit and implicit learning of event sequences: Evidence from event-related brain potentials. Journal of Experimental Psychology: Learning, Memory, \& Cognition, 22, 970-987.

Frensch, P. A., Buchner, A., \& Lin, J. (1994). Implicit learning of unique and ambiguous serial transactions in the presence and absence of a distractor task. Journal of Experimental Psychology: Learning, Memory, \& Cognition, 20, 567-584.

Frensch, P. A., Lin, J., \& Buchner, A. (1998). Learning versus behavioral expression of the learned: The effects of a secondary tonecounting task on implicit learning in the serial reaction time task. Psychological Research, 61, 83-98.

Frensch, P. A., \& Miner, C. S. (1994). Effects of presentation rate and individual differences in short-term memory capacity on an indirect measure of serial learning. Memory \& Cognition, 22, 95-110.

Grafton, S. T., Hazeltine, E., \& Ivry, R. [B.] (1995). Functional mapping of sequence learning in normal humans. Journal of Cognitive Neurosciences, 7, 497-510.

Heuer, H., \& Schmidtke, V. (1996). Secondary-task effects on sequence learning. Psychological Research, 59, 176-186.
Hsiao, A., \& Reber, A. S. (1998). The role of attention in implicit sequence learning. In M. A. Stadler \& P. A. Frensch (Eds.), Handbook of implicit learning (pp. 471-494). Thousand Oaks, CA: Sage.

Jiménez, L., Mendez, C., \& Cleeremans, A. (1996). Comparing direct and indirect measures of sequence learning. Journal of Experimental Psychology: Learning, Memory, \& Cognition, 22, 948-969.

Jolicoeur, P. (1999). Restricted attentional capacity between sensory modalities. Psychonomic Bulletin \& Review, 6, 87-92.

Keele, S. W., Ivry, R. B., Hazeltine, E., Mayr, U., \& Heuer, H. (1999). The cognitive and neural architecture of sequence representation: Timing in the SRT task (Tech. Rep. 98-03, Revised). Institute of Cognitive and Decision Sciences, University of Oregon.

MAYR, U. (1996). Spatial attention and implicit sequence learning: Evidence for independent learning of spatial and nonspatial sequences. Journal of Experimental Psychology: Learning, Memory, \& Cognition, 22, 350-364.

Nissen, M. J., \& Bullemer, P. (1987). Attentional requirements of learning: Evidence from performance measures. Cognitive Psychology, 19, 1-32.

PASHLER, H. E. (1998). The psychology of attention. Cambridge, MA: MIT Press.

RAH, S. K.-Y., Reber, A. S., \& Hsiao, A. T. (2000). Another wrinkle on the dual-task SRT experiment: It's probably not dual task. Psychonomic Bulletin \& Review, 7, 309-313.

Reber, P. J., \& SQuire, L. R. (1994). Parallel brain systems for learning with and without awareness. Learning \& Memory, 1, 217-229.

ReED, J., \& Johnson, P. (1994). Assessing implicit learning with indirect tests: Determining what is learned about sequence structure. Journal of Experimental Psychology: Learning, Memory, \& Cognition, 20, 585-594.

SchmidtKe, V., \& Heuer, H. (1997). Task integration as a factor in secondary-task effects on sequence learning. Psychological Research, 60, 53-71.

Shanks, D. R., \& Johnstone, T. (1998). Implicit knowledge in sequential learning tasks. In M. A. Stadler \& P. A. Frensch (Eds.), Handbook of implicit learning (pp. 533-572). Thousand Oaks, CA: Sage.

STADLER, M. A. (1989). On learning complex procedural knowledge. Journal of Experimental Psychology: Learning, Memory, \& Cognition, 15, 1061-1069.

STADLER, M. A. (1995). Role of attention in implicit learning. Journal of Experimental Psychology: Learning, Memory, \& Cognition, 21, 819-827.

Willingham, D. B., Nissen, M. J., \& Bullemer, P. (1989). On the development of procedural knowledge. Journal of Experimental Psychology: Learning, Memory, \& Cognition, 19, 1424-1430.

\section{NOTES}

1. When we first noticed this inconsistency, we contacted Steve Keele and his group to discover why they used the varying RSOA. Human memory is, indeed, wonderful. After several lengthy memorially challenged exchanges, Richard Ivry (personal communication, 1996) told us that he recalled being concerned that it might not be wise to allow subjects to get into a kind of "rhythm" by always having trials with the same temporal structure. Hence, the randomly varying RSOA was introduced. It was subsequently adopted by various other researchers (e.g., Frensch and co-workers) for the simple and commendable reason that they didn't wish to arbitrarily modify what was becoming "standard procedure" (Peter Frensch, personal communication, June 2000).

2. Above we reported the interaction between the good and poor counters and RSOA. A similar analysis between good counters and estimators yields a virtually identical interaction.

(Manuscript received November 10, 1999; revision accepted for publication June 5, 2000.) 